\title{
Probing the Dark Secrets of PNe with ORLs
}

\author{
X.-W. Liu \\ Department of Physics and Astronomy, University College London, \\ Gower Street, London WC1E 6BT, UK
}

\section{Introduction}

There are two long-standing problems in nebular astrophysics: a) The dichotomy of abundance determinations whereby heavy element abundances relative to hydrogen from collisionally excited lines (CELs) are systematically lower than those from (much fainter) optical recombination lines (ORLs), a discrepancy that can be traced back to Wyse (1942) and Aller \& Menzel (1945) who pioneered the ORL method; and b) The dichotomy of temperature determinations whereby $T_{\mathrm{e}}$ derived from the collisionally excited [O $\left.\mathrm{III}\right]$ forbidden line ratio is systematically higher than that derived from Balmer jump of the $\mathrm{H}$ I recombination spectrum, a discrepancy first discovered by Peimbert (1971) and attributed to temperature fluctuations in the nebula (Peimbert 1967). Thanks to the rapid progress in detector technology and recombination theory for multi-electron systems, significant progress has been made in recent years in our understanding of these two fundamental problems, which could potentially affect the derivation of abundances for a wide range of photoionized nebulae, both galactic and extragalactic. Both types of dichotomy are found to be real and are most likely caused by astrophysical effects rather than uncertainties in observations or in the basic atomic physics. The two problems are shown to be related and can be explained by assuming that PNe contain a small mass of extremely cold $\left(T_{\mathrm{e}} \sim 10^{3} \mathrm{~K}\right)$ inclusions, highly enriched in helium and heavy elements, embedded in diffuse material of "normal" abundances ( $\sim$ solar $)$ and temperature $\left(T_{\mathrm{e}} \sim 10^{4} \mathrm{~K}\right)$. The existence and nature of such $\mathrm{H}$-deficient inclusions in $\mathrm{PNe}$ remains a mystery. A recent review on this subject is given by Liu (2002).

\section{Observations of heavy element ORLs}

\subsection{Heavy element abundances from ORLs}

Liu et al. (1995) presented a detailed study of the rich ORL spectrum of NGC 7009 and showed that the C, N and O abundances derived from ORLs are all approximately a factor of five higher than the CEL values. Prompted by these findings, a deep optical spectroscopic survey of over a hundred Galactic $\mathrm{PNe}$ has been carried out. For many of them, ISO spectra from $2.4-197 \mu \mathrm{m}$ have also been obtained, which yield fine-structure line fluxes for many heavy element ions (Liu et al. 2001a). The optical and IR spectra, together with the archival $I U E$ data have been used to determine nebular thermal and density structures and elemental abundances, using both ORLs and CELs. Twelve PNe from this large data set are analyzed by Tsamis et al. (these proceedings). Dinerstein 
and Garnett observed two dozen PNe and detected O II ORLs in ten of them. Preliminary results are presented by Dinerstein et al. (2000) and Garnett \& Dinerstein (2001a). The discrepancy between the heavy element abundances derived from ORLs and CELs is found to vary from target to target, ranging from near agreement to over a factor of ten. Observational errors as the cause of the discrepancy can be ruled out (Mathis \& Liu 1999). So are blending by unknown lines or contamination of ORLs by excitation mechanisms other than recombination. For a given nebula, the discrepancies found for individual elements are of similar magnitude, consequently abundance ratios such as $\mathrm{C} / \mathrm{O}$, $\mathrm{N} / \mathrm{O}$ and $\mathrm{Ne} / \mathrm{O}$ are not affected (Liu et al. 1999; Luo, Liu \& Barlow 2001).

Our survey has led to the discovery of a handful of PNe with extremely rich and prominent ORL spectra, yielding ORL CNONe abundances that are often an order of magnitude higher than the corresponding CEL values. For example, in NGC 6153 (Liu et al. 2000) and in the bulge PN M 1-42 (Liu et al. 2001b), the ORL CNONe abundances are respectively a factor of 10 and 20 higher than those derived from CELs. Other examples of this rare group of PNe include: NGC 6778, whose rich O II ORL spectrum was first reported by Czyzak \& Aller (1973), Hf 2-2 and the bulge PNe M 2-36, M 3-26 and M 3-32. The strong C II $\lambda 4267$ emission in Hf 2-2 was first noted by Kaler (1988). In this most extreme nebula discovered so far, $\mathrm{O}$ II ORLs yield a record $\mathrm{O}^{2+} / \mathrm{H}^{+}$abundance ratio, 84 times higher than the CEL value (Liu et al., in preparation).

Nebulae with discrepancies over a factor of five are however rare, about $\sim 5 \%$ of the nebulae surveyed. Yet they are excellent laboratories to investigate the abundance determination problem and may simply represent a common phenomenon in its extreme, given that ORL abundances are invariably higher than CEL values, although in most cases by only a factor of two. Many ORLs from the abundant doubly ionized species of $\mathrm{C}, \mathrm{N}, \mathrm{O}$ and $\mathrm{Ne}$ have been detected. In addition to lines from the $3 \mathrm{p}$ and $3 \mathrm{~d}$ configurations, weaker transitions from higher levels such as $4 \mathrm{f}$ and $n \mathrm{~g}(n \geq 5)$ have also been detected. The $3 \mathrm{~d}-4 \mathrm{f}$ transitions, such as C II $\lambda 4267, \mathrm{~N}$ II $\lambda 4041, \mathrm{O}$ II $\lambda 4089$ and $\mathrm{Ne}$ II $\lambda 4392$, are particularly important. Arising from the highest- $J$ level of the $n=4$ configuration, these lines are highly hydrogenic, their strengths independent of the coupling scheme and immune to possible optical depth effects and fluorescence enhancement, and are therefore the most reliable abundance indicators. In essentially all cases, relative line intensities agree with the predictions of recombination theory. The large scatter reported by Dinerstein et al. (2000) in $\mathrm{O}^{2+} / \mathrm{H}^{+}$abundances derived from individual O II ORLs at the same nebular position, with the degree of scatter varying from target to target, is not seen in our data (Liu et al. 2001b). In one PN, M 1-42, fluorescence enhancement has been observed in 3p and $3 \mathrm{~d} N$ II triplet transitions, but not in singlet lines and triplet lines from the higher large angular momentum $4 \mathrm{f}$ levels (Liu et al. 2001b). The enhancement appears to be due to fluorescence by the stellar continuum rather than by the He I $1 \mathrm{~s}^{2}{ }^{1} \mathrm{~S}-1 \mathrm{~s} 8 \mathrm{p}{ }^{1} \mathrm{P}^{\mathrm{o}} \lambda 508.643$ resonance line proposed by Grandi (1976).

The $\mathrm{Mg}$ II $\lambda 44814 \mathrm{f}-3 \mathrm{~d}$ line is the strongest and easiest to measure ORL from any third-row ion. Barlow et al. (these proceedings) derived from this line $\mathrm{Mg} / \mathrm{H}$ abundances for ten $\mathrm{PNe}$, including four extreme PNe with very high ORL CNONe abundances, and found that they fall within a remarkably narrow range consistent with the solar value. Given that the depletion of magnesium onto 
dust grains is likely to be small, the result suggests that the large enhancements of ORL abundances over solar found in the same sample for second-row elements are due to astrophysical effects, rather than basic atomic physics.

\subsection{Spatial variations and correlations with other nebular properties}

Deep long-slit spectra have been used to map the $\mathrm{O}^{2+} / \mathrm{H}^{+}$abundance ratio distribution across the nebular surface using both $\mathrm{O}$ II ORLs and [O III] CELs for NGC 6153 (Liu et al. 2000), NGC 6720 (Garnett \& Dinerstein 2001b) and NGC 7009 (Luo \& Liu, these proceedings). In all cases, the ORL abundances are more strongly peaked towards the centre. In other words, the degree of discrepancy between the ionic abundances derived from these two types of emission line is also found to vary within a given nebula, and is generally largest close to the nebular centre. ORL abundances of $\mathrm{C}, \mathrm{N}$ and $\mathrm{Ne}$ are also found to show spatial variations similar to oxygen. In NGC 7009, in addition to the central peaking, a second peak in ORL abundances is observed at $\sim 18$ arcsec from the central star along the major axis, near a region of very low surface brightness, approximately half way between the bright shell and the two outlying ansae.

Garnett \& Dinerstein (2001a) find that the discrepancies between ORL and $\mathrm{CEL} \mathrm{O} \mathrm{O}^{2+} / \mathrm{H}^{+}$abundance ratios are greater for larger, lower-surface brightness $\mathrm{PNe}$ than for compact, dense ones. Similar trends are found amongst the $\mathrm{PNe}$ analyzed by Tsamis et al. (these proceedings), but with somewhat larger scatter.

\subsection{Balmer jump temperatures}

The discrepancy between ORL and CEL abundances has been found to be correlated with another long-standing discrepancy in nebular astrophysics: the dichotomy of $T_{\mathrm{e}}$ derived from the [O III] forbidden line ratio and from the hydrogen recombination Balmer jump (Peimbert 1971; Liu \& Danziger 1993). Peimbert (1971) attributed the difference between $T_{\mathrm{e}}([\mathrm{O} \mathrm{III}])$ and $T_{\mathrm{e}}(\mathrm{BJ})$ to temperature fluctuations and used the difference to make first-order corrections for the effects of temperature fluctuations on abundance determinations. Attempts to reproduce significant temperature fluctuations in PNe by photoionization modelling have been unsuccessful (e.g. Kingdon \& Ferland 1995; Gruenwald \& Viegas 1995). All extreme nebulae discovered so far having a very large ORL/CEL abundance ratio have a "typical" $T_{\mathrm{e}}([\mathrm{O} \mathrm{III}])$ between 8000 and $10000 \mathrm{~K}$, yet they all have a very low $T_{\mathrm{e}}(\mathrm{BJ})$. As the ORL/CEL ratio increases, $T_{\mathrm{e}}(\mathrm{BJ})$ decreases rapidly (Liu et al. 2001b). In the most extreme nebula Hf 2-2, where the ORL/CEL ratio reaches a record value of $84, T_{\mathrm{e}}(\mathrm{BJ})$ drops to $900 \mathrm{~K}$, a factor of ten lower than $T_{\mathrm{e}}([\mathrm{O} \mathrm{III}])=8820 \mathrm{~K}$ (c.f. Table 1 in $\left.\S 3.3\right)$. Clearly, the $T_{\mathrm{e}}(\mathrm{BJ})$ 's observed in these extreme $\mathrm{PNe}$ are so low that they cannot be explained by temperature fluctuations of the type envisaged by Peimbert $(1967,1971)$.

\section{Origin of the abundance an temperature determination dichotomy}

\subsection{Failure of the temperature/density fluctuation paradigm}

Various mechanisms have been proposed to explain the abundance and temperature determination dichotomy. Amongst these are temperature fluctuations (Peimbert 1971), density inhomogeneities (Rubin 1989; Viegas \& Clegg 1994) 
and abundance gradients (Torres-Peimbert et al. 1990). The determinations of ORL abundances for a number of elements, combined with the corresponding values deduced from CELs stretching from the UV to the far IR, have allowed these hypotheses to be tested quantitatively for the first time.

Liu et al. (2000) showed that the large discrepancies observed in NGC 6153 between the ORL and CEL abundances of $\mathrm{C}, \mathrm{N}, \mathrm{O}$ and Ne do not correlate with the excitation energy $E_{\text {ex }}$ nor critical density $N_{\text {crit }}$ of CELs - IR, optical or UV regardless. In particular, IR fine-structure lines, which have $E_{\text {ex }} \lesssim 10^{3} \mathrm{~K}$ and are therefore insensitive to temperature and temperature fluctuations, yield abundances similar to optical and UV CELs. Thus temperature fluctuations of the type envisaged by Peimbert (1967) as the cause of the discrepancies can be ruled out. Rubin et al. (these proceedings) and Wesson et al. (these proceedings) use WFPC2 images to map the $[\mathrm{O} \mathrm{III}]$ forbidden line temperature in NGC 7009 and NGC 6543, respectively, and find only small spatial variations in both PNe.

Similarly, the lack of dependence of the discrepancy on $N_{\text {crit }}$ argues against density inhomogeneities as the cause. For PNe containing ionized clumps with $10^{5} \lesssim N_{\mathrm{e}} \lesssim 10^{7} \mathrm{~cm}^{-3}, T_{\mathrm{e}}([\mathrm{O} \mathrm{III}])$ can be overestimated because the [O III] $\lambda \lambda 4959,5007$ lines are more severely suppressed in clumps than the $\lambda 4363$ line which has a higher $N_{\text {crit }}$, causing apparent temperature fluctuations (Viegas \& Clegg 1994). However, analysis of high order Balmer lines yields $N_{\mathrm{e}}=2000_{-1000}^{+2000}$ $\mathrm{cm}^{-3}$ in NGC 6153 (Liu et al. 2000). Since the ionized region is defined by $\mathrm{H}^{+}$ and, unlike forbidden density-diagnostic lines, $\mathrm{H}$ I lines cannot be collisionally suppressed in high density regions, the result shows that the nebula cannot contain a substantial amount of ionized gas in condensations with $N_{\mathrm{e}} \gtrsim 10^{5} \mathrm{~cm}^{-3}$.

\subsection{Chemically inhomogeneous two-component nebular models}

At $T_{\mathrm{e}} \sim 10^{4} \mathrm{~K}$, collisional excitation is several orders of magnitude more efficient than recombination. However, as $T_{\mathrm{e}}$ drops the emissivities of UV and optical CELs decrease exponentially, whereas those of ORLs increase. When $T_{\mathrm{e}}$ drops below $\sim 3000 \mathrm{~K}$, the emissivity of the O II $\lambda 4649 \mathrm{ORL}$ surpasses that of the [O III] $\lambda \lambda 4959,5007$ lines. Although the [O III] 52- and 88- $\mu \mathrm{m}$ fine-structure lines remain strong over a wider temperature range, they too become suppressed when $T_{\mathrm{e}}$ drops to only a few hundred $\mathrm{K}$. The IR lines can also be suppressed in high-density environments $\left(N_{\mathrm{e}} \gtrsim 10^{4} \mathrm{~cm}^{-3}\right)$, given their relatively low $N_{\text {crit }}$ 's.

The very low $T_{\mathrm{e}}(\mathrm{BJ})$ measured in those extreme PNe with very high ORL abundances suggests that there are at least two distinct emission regions in these nebulae, one of "normal" temperature $\left(T_{\mathrm{e}} \sim 10^{4} \mathrm{~K}\right)$ and "normal" abundances where the strong CELs originate; and another which is inferred to have very high heavy element abundances and a very low temperature (presumably due to enhanced cooling because of the high metallicity), which emits most of the observed flux from the heavy element ORLs but essentially no CEL emission. In this scenario, ORLs and CELs yield different element abundances and temperatures simply because they trace two completely different but intermingled ionized regions. Through ORLs, we are seeing a new nebular component, cold and enriched in heavy elements, hidden away from us in the light of strong CELs.

Abundance gradients as a possible cause of the high ORL abundances observed in PNe were first proposed by Torres-Peimbert et al. (1990) who showed 
that by assuming an inner C-rich zone in NGC 4361, the large discrepancy between carbon abundances deduced from ORLs and CELs can be reconciled. On the other hand, long-slit spectroscopy showed that the carbon ORL abundance is flat across NGC 4361 (Liu 1998). The observation however does not rule out the possibility of small $\mathrm{H}$-deficient inclusions well mixed with the diffuse gas.

Liu et al. (2000) showed that empirical models containing two components, each with its own $T_{\mathrm{e}}, N_{\mathrm{e}}$ and chemical composition, can account for many of the observed patterns in NGC 6153. One such model (IH2) has low density $\left(N_{\mathrm{e}} \sim 700 \mathrm{~cm}^{-3}\right), 500 \mathrm{~K}$ H-depleted material embedded in $N_{\mathrm{e}}=5500 \mathrm{~cm}^{-3}$, $T_{\mathrm{e}}=9500 \mathrm{~K}$ material with 'normal' abundances. An alternative model (IH3) has $4700 \mathrm{~K}$ high-density $\left(2 \times 10^{6} \mathrm{~cm}^{-3}\right)$, fully ionized, H-deficient knots embedded in the 'normal' component. In both models, the metal-rich component has a $\mathrm{He} / \mathrm{H}$ abundance ratio of $0.3-0.4$ and CNONe abundances which are $\sim 100$ times higher than those of the diffuse gas. In IH2 the IR fine-structure lines were suppressed by the very low $T_{\mathrm{e}}$ in the inclusions, whereas in IH3 the same effects were achieved by collisional de-excitation in the dense clumps. The filling factors of the $\mathrm{H}$-deficient inclusions in $\mathrm{IH} 2$ and $\mathrm{IH} 3$ are 0.3 and $3 \times 10^{-8}$, respectively. Thus in IH3 the H-deficient inclusions contain only a tiny amount of gas.

Photoionization models of NGC 6153 and M 1-42, incorporating H-deficient inclusions, have been constructed by Péquignot et al. (these proceedings; c.f. also Pequignot et al. 2002). Models of similar nature have also been calculated by Tylenda (these proceedings). These models yield an equilibrium $T_{\mathrm{e}} \sim 10^{3} \mathrm{~K}$ in the H-deficient component, compared to $\sim 10^{4} \mathrm{~K}$ in the ambient gas, and a density several times higher, so the two components are roughly in pressure equilibrium. In all cases, the H-deficient inclusions contain only $\sim 1 \%$ of the total mass and have helium and heavy element abundances similar to those derived from the empirical two-component models of Liu et al. (2000). The overall metallicity for the whole nebula is close to that of the ambient component ( solar). Ironically, the $\mathrm{He} / \mathrm{H}$ abundance, generally believed to be the best determined, is the least well constrained in such models, because all the strong He I and He II lines observable in the optical are ORLs. This uncertainty can be removed by measuring the He I $\lambda 10830$ line which is strongly enhanced by collisional excitation from the $2 \mathrm{~s}^{3} \mathrm{~S}$ metastable level (Péquignot et al., these proceedings).

\subsection{Testing the two-component nebular model}

The two-phase models, which postulate that there exist highly enriched low temperature islands within PNe, can be tested by direct imaging in both CELs and ORLs. This approach is however hampered by the weakness of ORLs and limited telescope angular resolutions. Depending on the sizes of the postulated $\mathrm{H}$-deficient inclusions, currently poorly constrained, and their surface brightness contrast against the ambient emission, they may never be spatially resolved. Fortunately, given the vast contrast in $T_{\mathrm{e}}$ between the two components, the models can also be tested (indirectly) if one can measure, by spectroscopy, the average $T_{\mathrm{e}}$ at which various lines are emitted. Specifically, the models predict

$$
T_{\mathrm{e}}(\mathrm{CNONe} \text { ORLs }) \lesssim T_{\mathrm{e}}(\mathrm{He} \mathrm{I}) \lesssim T_{\mathrm{e}}(\mathrm{BJ}) \lesssim T_{\mathrm{e}}(\mathrm{O} \text { III })
$$




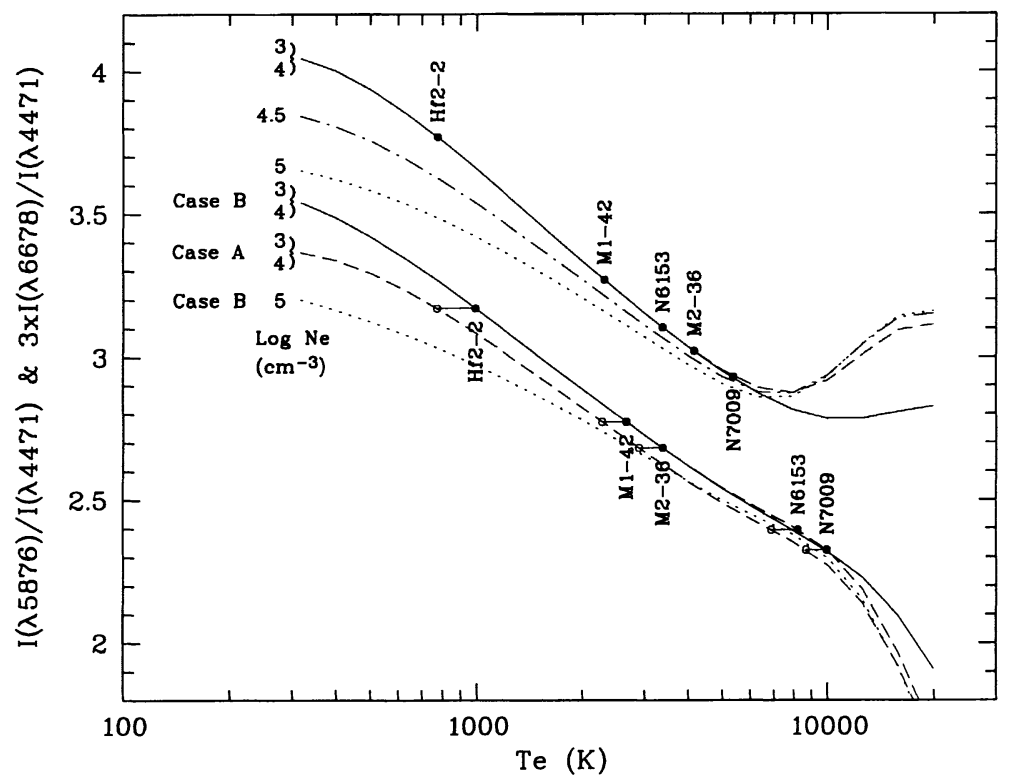

Figure 1. He I ORL ratios $\lambda 5876 / \lambda 4471$ (upper three curves) and $3 \times \lambda 6678 / \lambda 4471$ (lower three curves) as a function of $T_{\mathrm{e}}$ for $\log N_{\mathrm{e}}=3$, $4,5\left(\mathrm{~cm}^{-3}\right)$ (Smits 1996). For $3 \times \lambda 6678 / \lambda 4471$ and $\log N_{\mathrm{e}}=3,4$ $\left(\mathrm{cm}^{-3}\right)$, curves for both Case A and B recombination are shown.

with the difference between any two of these temperatures correlating with the ORL/CEL abundance ratio. For example, in the NGC 6153 model of Péquignot et al., about a few \% of $\mathrm{H}$ I emission, $\sim 30 \%$ of $\mathrm{He}$ I and essentially all of the heavy element ORLs arise from the cold metal-rich component, whereas optical/UV CELs are entirely dominated by the hot diffuse component (the situation for IR fine-structure lines is more complicated and depends on the $E_{\text {ex }}$ and $N_{\text {crit }}$ of the specific lines). The model predicts $T_{\mathrm{e}}([\mathrm{O} \mathrm{III}])=9100 \mathrm{~K}, T_{\mathrm{e}}(\mathrm{BJ})=7100 \mathrm{~K}$, $T_{\mathrm{e}}(\mathrm{He} \mathrm{I})=3600 \mathrm{~K}$ and $T_{\mathrm{e}}(\mathrm{CNONe}$ ORLs $)=1400 \mathrm{~K}$.

$T_{\mathrm{e}}(\mathrm{He} \mathrm{I})$ can be measured by observing the He I discontinuity at $3421 \AA$, produced by $\mathrm{He}^{+}$recombining to the $\mathrm{He} \mathrm{I} 2{ }^{3} \mathrm{P}^{\circ}$ level (Liu \& Danziger 1993). The discontinuity observed in NGC 6153 (Liu et al. 2000) is well fitted by the model of Péquignot et al. However, the $\mathrm{S} / \mathrm{N}$ was not sufficient to set an exact value, except a firm upper limit of $T_{\mathrm{e}}(\mathrm{He} \mathrm{I}) \lesssim 6000 \mathrm{~K}$. Better data are needed.

The intensity ratios of He I ORLs have some weak dependence on $T_{\mathrm{e}}$ which can be used to derive $T_{\mathrm{e}}$ (He I), provided accurate measurements can be obtained. The variations of He I $\lambda 5876 / \lambda 4471$ and $\lambda 6678 / \lambda 4471$ ratios as a function of $T_{\mathrm{e}}$ are plotted in Fig. 1, along with observed values in several extreme PNe (Liu et al. in preparation). $T_{\mathrm{e}}(\mathrm{He} \mathrm{I})$ derived from the $\lambda 5876 / \lambda 4471$ ratio is compared to $T_{\mathrm{e}}(\mathrm{BJ})$ and $T_{\mathrm{e}}([\mathrm{O} \mathrm{III}])$ in Table 1 . Also given in the Table are the ORL/CEL abundance ratios. The agreement of $T_{\mathrm{e}}(\mathrm{He} \mathrm{I})$ derived with the predictions of twocomponent photoionization models is remarkable. Note that the $\lambda 5876 / \lambda 4471$ ratio observed in $\mathrm{Hf} 2-2$ indicates that on average $\log N_{\mathrm{e}} \lesssim 4.5\left(\mathrm{~cm}^{-3}\right)$, i.e. no evidence of substantial high-density condensations with $N_{\mathrm{e}} \gtrsim 10^{5} \mathrm{~cm}^{-3}$. 
Table 1. Comparison of $T_{\mathrm{e}}(\mathrm{K})$ derived from the He I $\lambda 5876 / \lambda 4471$ ratio, the $\mathrm{H}$ I Balmer jump and from the [O $\mathrm{III}]$ forbidden line ratio

\begin{tabular}{lcccc}
\hline Nebula & $T_{\mathrm{e}}(\mathrm{He} \mathrm{I})$ & $T_{\mathrm{e}}(\mathrm{H}$ I BJ $)$ & $T_{\mathrm{e}}([\mathrm{O}$ III $])$ & ORL/CEL \\
\hline Hf 2-2 & 775 & 900 & 8820 & 84 \\
M 1-42 & 2310 & 3560 & 9220 & 22 \\
NGC 6153 & 3370 & 6080 & 9120 & 9.2 \\
M 2-36 & 4160 & 5900 & 8380 & 6.9 \\
NGC 7009 & 5380 & 8150 & 9980 & 4.7 \\
\hline \hline
\end{tabular}

Similarly, by resorting to the weak dependence of the heavy element ORL ratios on $T_{\mathrm{e}}$, one should obtain the true $T_{\mathrm{e}}$ in the H-deficient component. This is no easy task, as very accurate measurements are required for these extremely faint lines. Nevertheless the currently available data have already yielded some interesting results. The observed ratios of the strongest $\mathrm{O}$ II $3 \mathrm{~s}-3 \mathrm{p}$ line $\lambda 4649$ and $3 \mathrm{p}-3 \mathrm{~d}$ line $\lambda 4075$ to the strongest $3 \mathrm{~d}-4 \mathrm{f}$ line $\lambda 4089$ yield $200 \lesssim T_{\mathrm{e}}(\mathrm{ORL}) \lesssim 10^{3} \mathrm{~K}$ for the objects in Table 1. This removes a longstanding puzzle in determinations of $\mathrm{O}^{2+} / \mathrm{H}^{+}$using $\mathrm{O}$ II ORLs - when adopting the higher $T_{\mathrm{e}}(\mathrm{BJ})$ or $T_{\mathrm{e}}([\mathrm{O} \mathrm{III}]), \mathrm{O}^{2+} / \mathrm{H}^{+}$ratios derived from $3 \mathrm{p}-3 \mathrm{~d}$ and $3 \mathrm{~d}-4 \mathrm{f}$ transitions are systematically higher by $\sim 60 \%$ than those yielded by the $3 \mathrm{~s}-3 \mathrm{p}$ lines (Liu et al. $1995,2000,2001 b)$. On the other hand, the weaker transitions, 3p-3d $\lambda 4072$ and $3 \mathrm{~s}-3 \mathrm{p} \lambda 4415$, appear to be too strong by $\sim 0.1-0.2$ dex compared to $\lambda 4089$, yielding higher $T_{\mathrm{e}}(\mathrm{ORL})$ 's. While better data are needed, one possibility is that the $2 \mathrm{p}^{2}{ }^{3} \mathrm{P}_{2}$ level of the recombining $\mathrm{O}^{2+}$ is underpopulated relative to its statistical equilibrium value. If this is the case, then it can potentially provide a direct method to estimate $N_{\mathrm{e}}$ in the H-deficient component, thus its filling factor and mass, once the relevant atomic data are available.

In short, there is strong evidence that He I and heavy element ORLs do arise from environments of very low $T_{\mathrm{e}}$, supporting the two-phase nebular models.

\subsection{Nature and origin of the H-deficient inclusions}

A small number of PNe, such as A 30 and A 78 are known to contain H-deficient knots (Jacoby \& Ford 1983), and are interpreted by Iben et al. (1983) as "bornagain" PNe. Strong ORLs from CNONe ions have been observed from such knots (Wesson et al., these proceedings). However not all PNe in Table 1 have a $\mathrm{H}$-deficient CS. Moreover, the fact that the postulated $\mathrm{H}$-deficient inclusions are O-rich and the fact that they have $\mathrm{C} / \mathrm{O}, \mathrm{N} / \mathrm{O}$ and $\mathrm{Ne} / \mathrm{O}$ ratios similar to the diffuse component, are incompatible with any known nuclear processes (Liu et al. 2000) and are difficult to explain in the "born-again" scenario.

Could the H-deficient inclusions come from evaporation of the planetary system (planets, asteroids, comets, etc) of the progenitor star? Given that the postulated H-deficient inclusions contain only a few Jupiter masses, this may not be so exotic as it sounds. After all, many stars are now known to harbour planets. If so, it may also provide an explanation for the high ORL/CEL abundance ratios observed in some $\mathrm{H}$ II regions (e.g. Tsamis et al. this volume). 
Finally, Hf 2-2 has a close binary central star (Lutz et al. 1998) and shares many spectral characteristics, e.g. a large Balmer jump and strong heavy element ORLs, with old novae such as DQ Her (Williams et al. 1978). Possible links between these two classes of object are therefore worth further investigation.

Acknowledgments. I thank Drs. M. J. Barlow, I. J. Danziger, S.-G. Luo, C. Morisset, D. Péquignot, R. Rubin, P. J. Storey, and Mr. Y. G. Tsamis and R. Wesson for the fruitful collaboration with them on this interesting subject.

\section{References}

Aller, L. H., Menzel, D. H. 1945, ApJ, 102, 239

Czyzak, S. J., \& Aller, L. H. 1973, ApJ, 181, 817

Dinerstein, H. L., Lafon, C. P., \& Garnett, D. R. 2000, in Asymmetric Planetary Nebulae II, ed. J. Kastner, et al. (San Francisco: ASP), 301

Garnett, D. R., \& Dinerstein, H. L. 2001a, Rev.Mex.Astron.Astrofis. Ser.de Conf., 10, 13

Garnett, D. R., \& Dinerstein, H. L. 2001b, ApJ, 558, 145

Gruenwald, R., \& Viegas, S. M. 1995, A\&A, 303, 535

Grandi, S. A. 1976, ApJ, 206, 658

Iben, I., Jr., Kaler, J. B., Truran, J. W., \& Renzini, A. 1983, ApJ, 264, 605

Jacoby, G. H., \& Ford, H. C. 1983, ApJ, 266, 298

Kaler, J. B. 1988, PASP, 100, 620

Kingdon, J., \& Ferland, G. J. 1995, ApJ, 450, 691

Liu, X.-W. 1998, MNRAS, 295, 699

Liu, X.-W. 2002, Rev.Mex.Astron.Astrofis. Ser.de Conf., in press

Liu, X.-W., et al. 1999, in Chemical Evolution from Zero to High Redshift, ed. J. R. Walsh \& M. Rosa (Berlin: Springer-Verlag), 39

Liu, X.-W., \& Danziger, I. J. 1993, MNRAS, 263, 256

Liu, X.-W., Barlow, M. J., Cohen, et al. 2001a, MNRAS, 323, 343-361

Liu, X.-W., Luo, S.-G., Barlow, et al. 2001b, MNRAS, 327, 141

Liu, X.-W., Storey, P.J., Barlow, M.J., \& Clegg R.E.S. 1995, MNRAS, 272, 369

Liu, X.-W., Storey, P. J., Barlow, M. J., et al. 2000, MNRAS, 312, 585

Luo, S.-G., Liu, X.-W., \& Barlow, M. J. 2001, MNRAS, 326, 1049

Lutz, J., Alves, D., Becker, A., et al. 1998, AAS, 192, 5309

Mathis, J. S., \& Liu, X.-W. 1999, ApJ, 521, 212

Peimbert, M. 1967, ApJ, 150, 825

Peimbert, M. 1971, Bol. Obs. de Tonantzintla Y Tacubaya, 6, 29

Péquignot, D., Amara, M., Liu, X.-W., et al. 2002, Rev.Mex.Astron.Astrofis. Ser.de Conf., in press

Rubin, R. H. 1989, ApJS, 69, 897

Smits, D. P. 1996, MNRAS, 278, 683

Torres-Peimbert, S., Peimbert, M., \& Peña, M. 1990, A\&A, 233, 540

Viegas, S., \& Clegg, R. E. S. 1994, MNRAS, 271, 993

Williams, R. E., Woolf, N. J., Hege, E. K., et al. 1978, ApJ, 224, 171

Wyse, A. B. 1942, ApJ, 95, 356 